\title{
A Taylor Vortex Photocatalytic Reactor for Water Purification
}

\author{
Tapan K. Sengupta, ,, \\ Department of Mechanical Engineering and Department of Chemical and Environmental Engineering, \\ The National University of Singapore, 10 Kent Ridge Crescent, Singapore 119260
}

\begin{abstract}
A detailed analysis has been performed for a heterogeneous photocatalytic Taylor vortex reactor that uses flow instability to recirculate fluid continually from the vicinity of the rotating inner cylindrical surface to the stationary outer cylindrical surface of an annulus. In the present research, a detailed timeaccurate computation shows the different stages of flow evolution and the effects of the finite length of the reactor in creating eddies, which results in a very high overall efficiency of photocatalytic conversion. The physical arrangement consider ed is such that pollutant degradation is maximized by the motion of fluid particles in a specific regime of centrifugal instability. Also provided are detailed flow structures for the chosen parameters when the reactor is started impulsively.
\end{abstract}

\section{Introduction}

Semiconductor photocatalysis is an advanced oxidation technology for water purification that has recently been reviewed by Chen et al. ${ }^{1}$ Heterogeneous photocatalytic technol ogy couples low-energy ultraviolet light with semiconductors acting as photocatalysts to overcome many of the drawbacks that exist for traditional water treatment methods. Using this technology, in situ degradation of toxic organic compounds can be achieved by oxidation (using the photogenerated holes 2,3 ) and removal of toxic metal ions by reduction (using photogenerated electrons ${ }^{4}$ ). The appeal of this process technology is the prospect of complete mineralization of pollutants to environmentally harml ess compounds. In addition, the process uses atmospheric oxygen as the oxidant, and the catalyst (usually $\mathrm{TiO}_{2}$ ) is cheap, stable and nontoxic and can be used for extended periods without substantial loss of activity. Moreover, the technique employs very low energy UV-A light $(\lambda<380$ $\mathrm{nm})$, resulting in energy requirements as low as $1-5$ $\mathrm{W} / \mathrm{m}^{2}$ of catalyst surface area, and more importantly, it can even be activated even by sunlight. ${ }^{5}$

Despite the potential of this promising technology, the devel opment of a practical water treatment system has not yet been successfully achieved as a result of various limitations. ${ }^{6}$ There are several factors that impede the efficient design of photocatalytic reactors. In this type of reactor, in addition conventional reactor complications such as reactant-catalyst contact, flow patterns, mixing, mass transfer, reaction kinetics, catalyst installation, temperature control, etc., an additional engineering factor related to illumination of catalyst becomes relevant. The illumination factor is of utmost importance because the amount of catalyst that can be activated determines the water treatment capacity of the reactor. The high degree of interaction between transport processes, reaction kinetics, and light absorption leads to a strong coupling of physicochemical

* Corresponding author. Tel.: + 65874 8049. Fax: + 65 779 1936. E-mail: cheakr@nus.edu.sg.

† Department of Mechanical Engineering.

‡ On leave from Indian Institute of Technology, Kanpur 208016, India.

$\S$ Department of Chemical and Environmental Engineering. phenomena and is one of the major hurdles in the technical development of a photocatalytic reactor.

Photocatalytic reactions are promoted by solid photocatalyst particles that usually constitute a discrete phase distributed within a continuous fluid phase in the reactor. Therefore, at least two phases, i.e., liquid and solid, are present in the reactor. The solid phase could be dispersed as a slurry ${ }^{2}$ or could be stationary ${ }^{3}$ within the reactor. However, the use of suspensions requires the separation and recycling of the ultrafine (submicronsized) catalyst from the treated liquid and can be an inconvenient, time-consuming, and expensive process. In addition, the depth of penetration of UV light is limited because of strong absorption by both catalyst particles and dissolved pollutants. The above problems could be avoided in a fixed-bed photoreactor in which photocatalyst particles are immobilized onto a fixed transparent surface, such as the reactor wall. However, immobilization of catalyst on a support generates a unique problem. The reaction occurs at the liquid-solid interface, and usually, only part of the catalyst is in contact with the reactant. Hence, the overall rate is limited by the mass transport of the pollutant to the catalyst surface. ${ }^{7,8} \mathrm{In}$ addition, the overall rate of reaction is usually slow compared to conventional chemical reaction rates because of the low concentration level of the pollutant.

The scale-up of fixed-bed photocatalytic reactors has been severely limited because reactor configurations have not been able to address the issue of mass transfer of pollutants to the catalyst surface. The new reactor design concepts must deal with this challenge. Earlier experimental studies of our group on catalyst-coated tube bundles, ${ }^{9}$ novel immersion-type lamps, ${ }^{10}$ and rotating tube bundles reveal ed that photocatalytic reactions are mainly diffusion- (mass-transfer-) controlled. In our earlier studies, we enhanced mass transfer by increasing the mixing of fluids through turbulence and the introduction of baffles. ${ }^{7}$ In this work, a new reactor concept is developed that uses flow instability to increase the reaction yield throughout the reactor volume.

Sczechowski et al. ${ }^{11}$ reported their experimental studies related to enhancing the photoefficiency of a Taylor vortex reactor (TVR). A schematic of the reactor is shown in Figure la. The toroidal vortices that are 


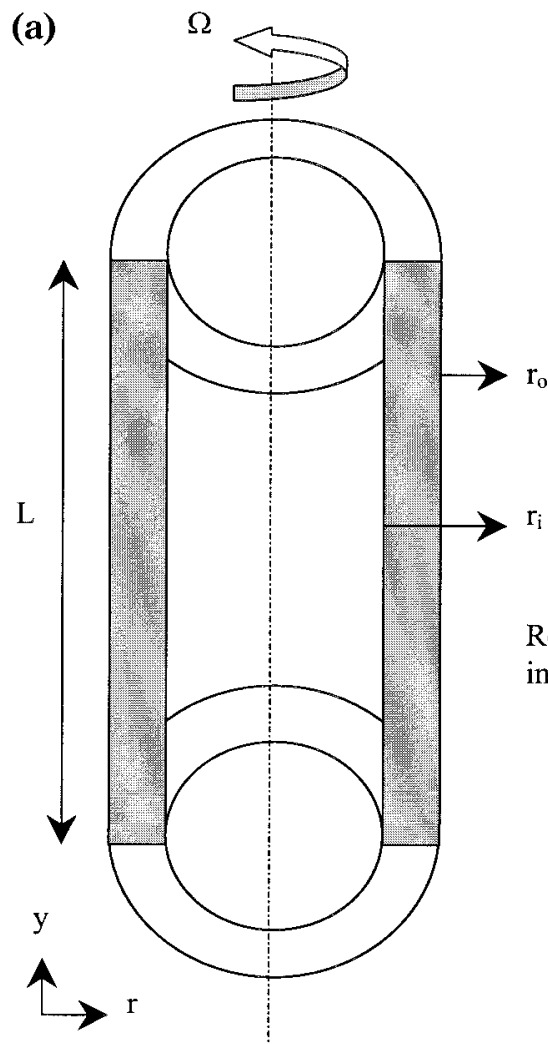

(b)

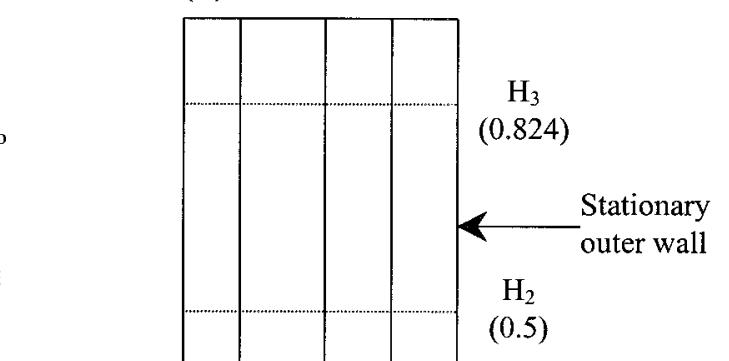

Figure 1. (a) Schematic diagram of the Taylor vortex reactor and (b) location of the observation stations in the annular region to analyze flow behavior. $d^{*}=0$ (rotating inner cylinder), $d^{*}=1$ (stationary outer wall), $y^{*}=0$ (bottom), $y^{*}=1$ (top).

usually formed in the annular gap between the cylinders are shown in Figure 2a. The nonwavy vortex flow appears as a consequence of primary instability given by appropriate nondimensional numbers such as the Taylor number and Reynolds number. Assuming the annular gap $d=\left(r_{0}-r_{i}\right)$ and the rotation rate of cylinder $\Omega$, the Taylor number is defined as

$$
\mathrm{Ta}=\frac{\mathrm{r}_{\mathrm{i}} \mathrm{d}^{3}\left[\Omega_{\mathrm{i}}^{2}-\Omega_{\mathrm{o}}^{2}\right]}{v^{2}}
$$

When the annular gap, $d$, is small compared to the inner cylinder radius with very large aspect ratios of the geometry (i.e., very large lengths) and $0 \leq \Omega_{0} / \Omega_{\mathrm{i}} \leq 1$, Taylor ${ }^{12}$ showed that the primary instability in the form of the appearance of Taylor vortices occurs at Tacrit $\approx$ 1708. When the Reynolds number, defined in terms of the gap width as the length scale and the surface velocity on the inner cylinder surface as the velocity scale, is increased to somewhat higher values, one sees wavy vortex flow, also shown in Figure $2 \mathrm{a}$, which is characterized by the appearance of circumferential and axial wavy motion of the toroidal vortices. Sczechowski et al. ${ }^{11}$ observed that, when catalyst particles were used as a slurry, the useful reaction took place only periodically when the fluid was in contact with the illuminated inner cylinder surface. They reported a 3-fold increase of the photoefficiency when the reactant was illuminated for less than $150 \mathrm{~ms}$, when it stayed in the dark for more than $1 \mathrm{~s}$. The maximum photoefficiency they could achieve was $30 \%$ at a rate of $300 \mathrm{rpm}$ for the inner cylinder when $10 \mathrm{~g} / \mathrm{L}$ loading of $\mathrm{TiO}_{2}$ was used. The major problem of achieving higher photoefficiencies was related to the transport of purified fluid from the vicinity of the catalyst. However, use of the catalyst as a slurry raises the problem of separating submicron-sized catalyst particles after the purification stage. Moreover, the working fluid is optically dense, and ther efore, the light penetration depth is restricted to a distance that is on the order of the boundary layer thickness of the inner cylinder. In view of all of these factors, we have considered in our present work a TVR of similar geometry, but instead of a slurry-type reactor, the photocatalyst was assumed to be immobilized (fixed) on the outer surface of the inner cylinder and a fluorescent lamp was assumed to illuminate the inner cylinder. Thus, one can use a very low level of catalyst loading and, simultaneously, eliminate the process of separating the catalyst particles after the purification stage. Enhanced purification can be obtained by utilizing the fluid dynamical instability associated with the centrifugal instability in the cylindrical annular geometry. It is essential to focus on the twin aspects, photocatalysis and centrifugal instability, in an annular cylindrical geometry.

\section{Taylor-Couette Flow and Flow Instability}

The laminar flow confined within the annulus region between two coaxial cylinders, with the inner one differentially rotating with respect to the outer, suffers centrifugal instability depending on the geometry (aspect ratio) and rotation rates. The criteria of centrifugal instability was first enunciated by Rayleigh, 13 who showed that an inviscid rotating flow is unstable if the energy of rotation associated with the fluid particle decreases radially outward. Under such unstable configurations, one notices the appearance of circumferential toroidal vortices between the two cylinders, which are known as Taylor-Couette vortices. Tayl or ${ }^{12}$ was the first to report experimental verification of this phenom- 
(a)

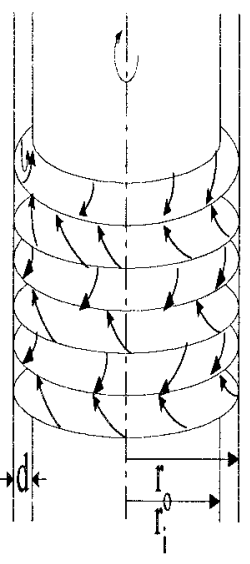

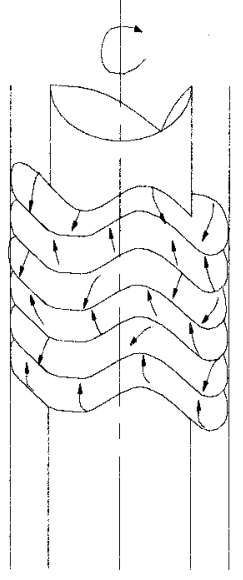

(b)



(c)

Outer Cylinder

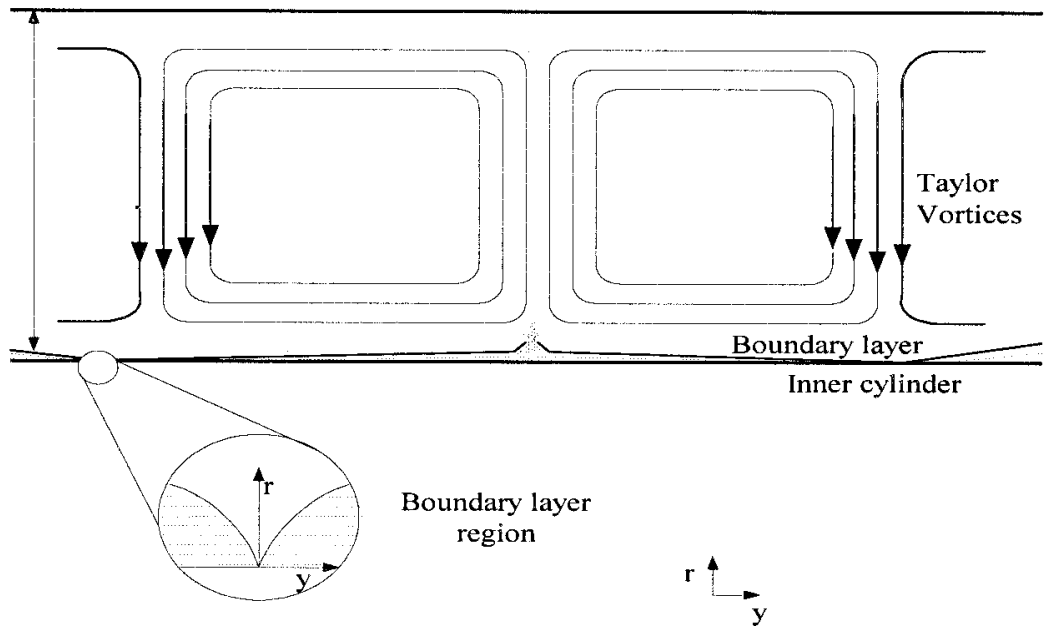

Figure 2. (a) Vortices without and with circumferential waves, (b) schematic presentation of streamlines in vortical cells, and (c) radial mass transfer in Taylor-Couette flow.

enon. These vortices evolve because of the adverse gradient of angular momentum that creates a potential unstable arrangement of flow (see ref 14). Such an unstable condition arises naturally if the outer cylinder is held stationary while the inner cylinder is rotated at a sufficiently high rotation rate-an arrangement considered in this paper. In such an unstable condition, the annulus is filled with a pair of counter-rotating vortices. This can be viewed in Figure $2 b$ and $c$. Figure $2 c$ shows the formation of a boundary layer on the inner surface. Moreover, it shows that the boundary layer oscillates periodically between almost zero thickness to a maximum between the counter-rotating vortex pair, where the two shear layers approaching each other spew out a jet of fluid toward the outer wall. The fluid particles in their motion around the toroidal vortices come periodically into contact with the inner surface where the catalyst is immobilized, and in the presence of light, the catalyst is activated, resulting in the occurrence of the redox reaction. The residence time of the particles in the illuminated region is thus a function of the angular velocity of the recirculating vortex as well as the size of the vortex. The latter, once again, depends on the gap size and the number of vortices formed in a given length of the reactor.
However, the situation can be different if the Taylor vortices are established in a reactor of short length. Experiments carried out by Burkhalter and Koschmieder ${ }^{15}$ have revealed that the axial wavel ength of the Taylor vortices for finite cylinders are different from those predicted by stability theory for singly periodic Taylor vortices in infinitely long cylinders. F urthermore, the effective wavelength al so depends on the end-cap boundary conditions. Andereck et al. ${ }^{16}$ pointed out that there could be many flow states, which could be distinguished by their symmetry under rotation and reflection, i.e., their azimuthal and axial wavenumbers. This has been attributed to the existence of multiple stable solutions of the governing NavierStokes equation for flows far from equilibrium.

Werel ey and Lueptow ${ }^{17}$ showed experimentally that wavy vortex flow, characterized by both axial and radial azimuthal deformation of the vortices, became stronger, and the outflow between pairs of vortices became jetlike. According to their findings, significant transfer of fluid between neighboring vortices occurs in a cyclic fashion at certain points along an azimuthal wave so that, while one vortex grows in size, the two adjacent vortices become smaller, and vice versa. This aspect of the flow is important, because traditionally, it is assumed that 
Table 1. Geometric Dimensions of the Two Reactor Configurations Considered

\begin{tabular}{lll}
\hline \multicolumn{1}{c}{ specification } & configuration 1 & configuration 2 \\
\hline length, $\mathrm{L}, \mathrm{m}$ & 0.4245 & 0.4245 \\
inner radius, $\mathrm{r}_{\mathrm{i}}, \mathrm{m}$ & 0.0434 & 0.0434 \\
outer radius, $\mathrm{r}_{0}, \mathrm{~m}$ & 0.0523 & 0.0545 \\
annular gap, d, m & 0.0089 & 0.0111 \\
aspect ratio, $\mathrm{L} / \mathrm{d}$ & 47.70 & 38.24 \\
rotation speed, $\Omega, \mathrm{rad} / \mathrm{s}(\mathrm{rpm})$ & $0.655(6.25)$ & $0.524(5.0)$ \\
volume of liquid treated, $\mathrm{V}_{\mathrm{L}}, \mathrm{m}^{3}$ & $1.136 \times 10^{-3}$ & $1.449 \times 10^{-3}$ \\
Reynol ds number, $\mathrm{Re}$ & 253 & 253 \\
Taylor number, $\mathrm{Ta}$ & 13126 & 16298
\end{tabular}

two Taylor-Couette vortices making up a pair are independent of each other and of the same size, and it is customary to treat this as plug flow. If, indeed, significant mass transfer occurs between adjacent vortex pairs, then it can be used for additional benefit in the present application for performance enhancement of the reactor. It has also been reported that the degree of transfer of the fluid is greater for $\mathrm{Re}=253$ than for either higher or lower Reynolds numbers. In addition to flow in to and out of adjacent vortices taking place periodically, the individual vortex centers also move both axially and radially in a cyclic fashion. Once again, the maximum departure in the radial direction is a strong function of the Reynol ds number and is found to be a maximum for $\mathrm{Re}=253$. It is for these reasons that we have decided to investigate two geometries (aspect ratios) that correspond to this optimum Reynol ds number. However, to avoid long computational times, and also for practical utility, the establishment of flow is considered to occur in an impulsive fashion. In a practical design, one would like to have the purification process completed in a limited time.

\section{Computational Details}

In computing theflow, the three-dimensional NavierStokes equation is solved in a primitive formulation using the commercial software Fluent. This approach was found to be adequate as the flow considered was laminar and could thus be computed without the need for resolving large ranges of wavenumbers and circular frequencies. In solving the governing equations, no simplifications were made regarding symmetry or reflection of the solution. In addition, the a priori steadystate solution was not assumed; rather, the timeaccurate solution was calculated. We considered two different geometric dimensions to study the effect of the annular gap for a constant Reynolds number of 253, the optimum Reynolds number observed experimentally by Werely and Lueptow. ${ }^{17}$ The dimensions of the two reactors considered in this study are given in Table 1 . Reactor configuration 1 is identical in its dimensions to the reactor of Werely and Lueptow ${ }^{17}$ and is referred to as the reference configuration. The main intention of this study is to show the efficacy of the immobilized photocatalytic reactor for the chosen parameter values. In reactor configuration 2, the annular gap (d) is increased for a constant reactor length, to increase the amount of liquid that can be treated (i.e., the capacity) inside the reactor. To be able to compare the results at same Reynol ds number $(\operatorname{Re}=253)$, the rotation speed, $\Omega$, was reduced. When $\mathrm{d}$ was increased to $0.0111 \mathrm{~m}$ (configuration 2), the rotation speed had to be lowered to $5 \mathrm{rpm}$ to maintain the Reynolds number at 253 . This effectively increases the capacity of the reactor by $27.6 \%$,
Table 2. Rate Expressions for Phenol and SBB Dye Used in This Study

$$
\begin{aligned}
& \text { phenol }^{3} \quad \mathrm{R}=-\frac{\mathrm{dC}_{\mathrm{s}}}{\mathrm{dt}}=\mathrm{k}_{0} \exp \left[-\frac{\mathrm{E}}{\mathrm{RT}}\right] \mathrm{I}^{\beta}\left[\frac{\mathrm{K}_{\mathrm{O}_{2}} \mathrm{P}_{\mathrm{O}_{2}}}{1+\mathrm{K}_{\mathrm{O}_{2}} \mathrm{P}_{\mathrm{O}_{2}}}\right]\left[\frac{\mathrm{K}_{\mathrm{s}} \mathrm{C}_{\mathrm{s}}}{1+\mathrm{K}_{\mathrm{s}} \mathrm{C}_{\mathrm{s}}}\right] \\
& \text { where } \quad \mathrm{k}_{0}=1.19 \times 10^{-4} \mathrm{~mol} \mathrm{~m} \mathrm{~m}^{-2} \mathrm{~s}^{-1}, \mathrm{E}=11.80 \mathrm{~kJ} / \mathrm{mol} \\
& \beta=0.82, \mathrm{~K}_{\mathrm{O}_{2}}=12.71 \mathrm{~atm}^{-1}, \mathrm{~K}_{\mathrm{S}}=4.26 \mathrm{mM}^{-1} \\
& \text { SBB dye } \mathrm{S}^{5}=-\frac{\mathrm{dC}_{\mathrm{s}}}{\mathrm{dt}}=\left[\frac{\mathrm{k}(\mathrm{l}, \mathrm{w}) \mathrm{KC}_{\mathrm{s}}}{1+\mathrm{KC}_{\mathrm{s}}}\right] \\
& \text { where } \\
& k(I, w)=\frac{\left.k_{s} a^{\prime}(\sqrt{w})\right|^{b}}{1+a^{\prime}(\sqrt{w}) I^{b}} \\
& \mathrm{k}_{\mathrm{s}}=0.38 \mathrm{mmol} / \mathrm{m}^{2} / \mathrm{s} \\
& a^{\prime}=3.45, b=0.85, K=18.5 \mathrm{~m}^{3} / \mathrm{mol}
\end{aligned}
$$

while reducing the energy cost (lower power required to maintain lower rpm, $\Omega$ ).

Two different model pollutants were considered in this work, namely, phenol and SBB dye. These two model compounds were studied by our group earlier and will hel p us to study the effect of reaction rate constants on photocatalytic degradation. Because the initial concentrations of pollutants are usually very low (100 ppm), it is expected that the chemical reaction will not influence the fluid dynamic behavior of the system. This was observed to be the case, as shown later. The photocatalytic degradation rates for phenol and SBB dye were calculated using the equations given in Table 2.

The density of the system was assumed to be constant and equal to that of water, as both of the pollutants are present in trace amount. The various locations where the solutions were investigated and plotted are shown in Figure 1b. The boundary conditions that are applied on the inner and outer cylindrical surface correspond to no-slip conditions. The end caps are considered to be a part of the outer cylinder and hence are stationary.

For generating grids within the annulus region of the geometry, every edge in three directions was defined by certain nodes. There are high-shear regions near the inner cylinder wall, the outer cylinder wall, and the endcap regions. Therefore, axial and radial derivatives of all physical variables across such layers are larger than the corresponding derivatives in the azimuthal direction, and consequently, more grid points are taken in the axial and radial directions compared to the azimuthal direction. These extragrid points are incorporated by being more clustered at the two ends and next to the inner and outer walls. To enhance the direct mapping of the grid from the upper wall to the bottom wall, the total geometry was separated into two volumes by a brick. Two interior planes were created by this process within the annular space that was used to analyze our results at the time of postprocessing. To obtain the instability pattern of flow in annular area, both a finer grid and a coarser grid were chosen to analyze the grid dependency of results. Grid points along the three directions were taken as follows: $r \times \theta$ $\times \mathrm{h}=200 \times 60 \times 150$ in the fine-grid case and $100 \times$ $40 \times 75$ in the coarse-grid case. The Fluent preprocessor GAMBIT 1.1 was used to create the geometry and generate the grid for both cases. Complete descriptions of the mesh volumes and nodes generated for the simulations are as follows:

For the finer grid the mesh volume was 1.8 million with a total of 1821060 nodes, and the total number of elements was once again 1.8 million. Creation of this grid required a total of 393.66 CPU seconds with 431.2 MB of memory. For the coarser grid, there were 0.3 
5272 Ind. Eng. Chem. Res., Vol. 40, No. 23, 2001

(a) Coarse grid simulation $(\mathrm{t}=10 \mathrm{~s})$

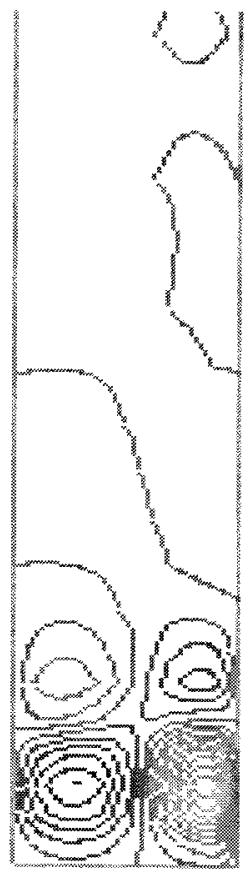

(c) Coarse grid simulation $(\mathrm{t}=30 \mathrm{~s})$

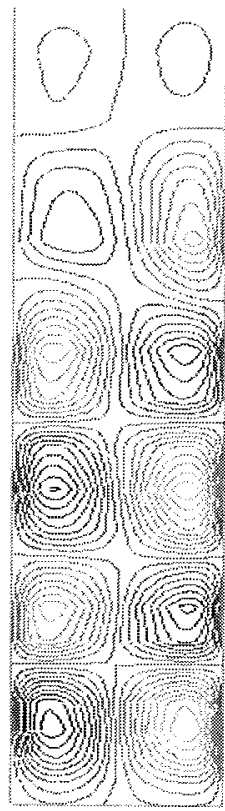

(b) Fine grid simulation $(t=10 \mathrm{~s})$

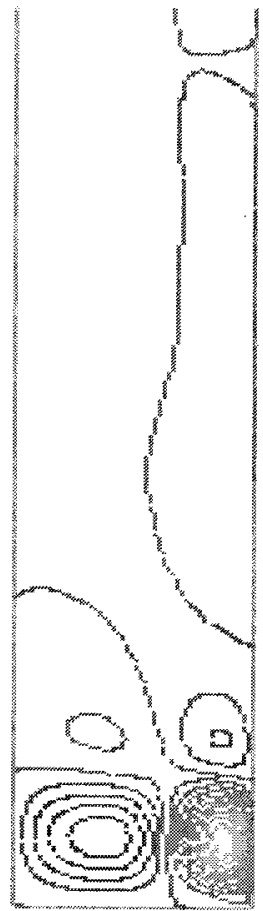

(d) Fine grid simulation $(t=30 \mathrm{~s})$

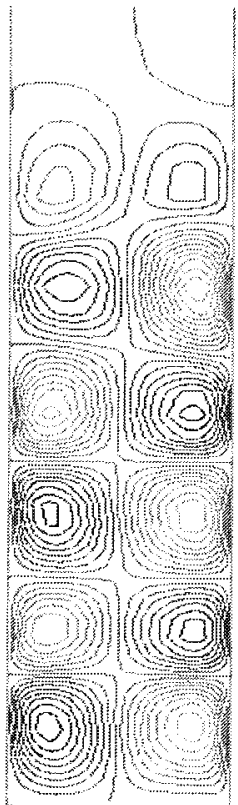

Figure 3. Grid convergence is shown by the contour of the axial velocity (bottom $11.78 \%$ of the reference reactor is shown). The right boundary is the rotating inner wall $\left(d^{*}=0\right)$.

million cells, 907000 total quadrilateral faces (3000 inner wall faces, 11000 outer wall spaces, 15000 interior plane faces, and 878000 other interior plane faces). The total number of nodes was 307040.

The present set of computations of the Navier-Stokes equation for Taylor-Couette geometry is expensive. For the finer grid, the random access memory (RAM) requirement is $1.3 \mathrm{~GB}$, which demands a large computing machine. The case with reaction (flow plus reaction) was submitted to a 1.5-GB RAM Windows NT workstation, and the case without reaction was submitted to a mainframe supercomputer (SGI origin 2000). For the coarse grid, the CPU requirement was considerably less, as the number of grids was $1 / 6$ that of the finer one.

\section{Results and Discussion}

Flow E volution. It has al ready been mentioned that the performance of the reactor depends on the photoefficiency of the photocatalytic process, as well as on the mass transfer efficiency of the fluid from the vicinity of the rotating inner cylinder to the stationary outer cylinder and back within a single Taylor-Couette vortex. The latter depends on the primary instability 

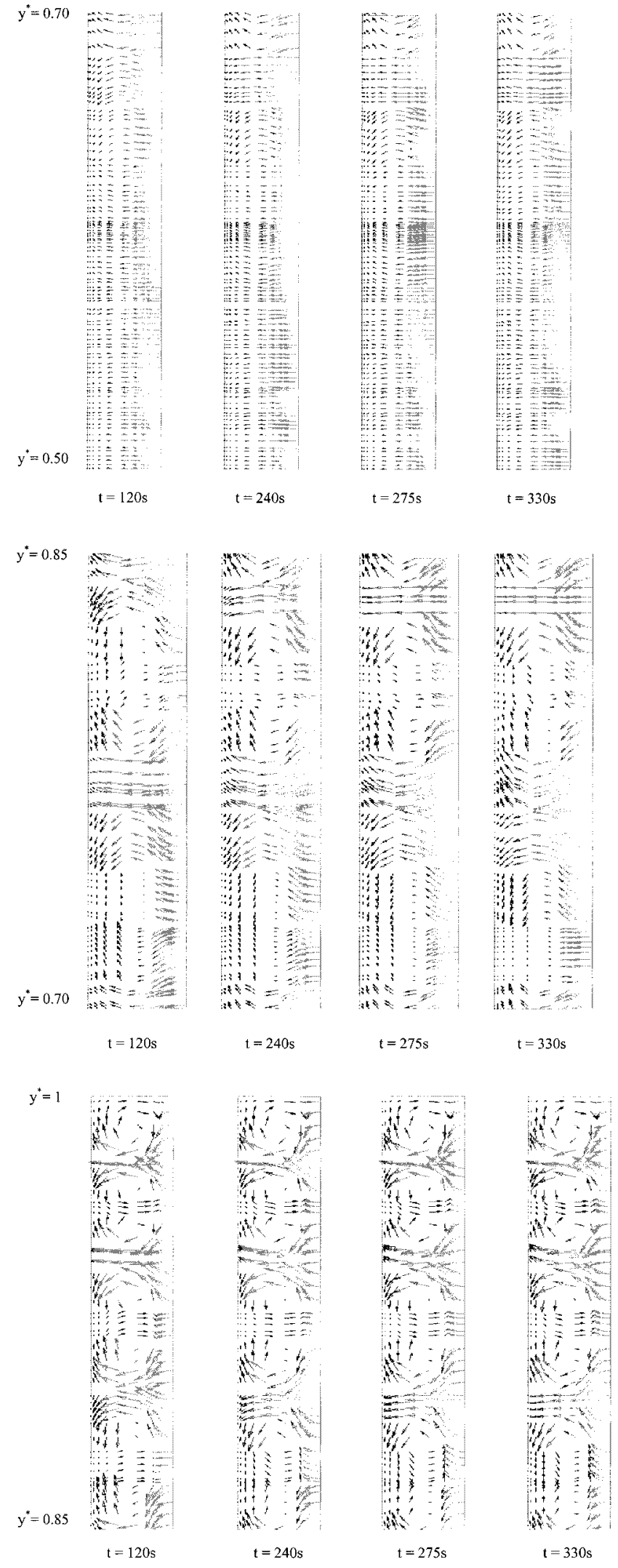

Figure 4. (a) Velocity vectors shown in the radial plane for $y^{*}=$ $0.5-0.7$ at various times. The right side indicates the rotating inner wall $\left(d^{*}=0\right)$. (b). Velocity vectors shown in the radial plane for $y^{*}=0.70-0.85$ at different times. The right side indicates the rotating inner wall $\left(d^{*}=0\right)$. (c). Velocity vectors in the radial plane for $\mathrm{y}^{*}=0.85-1$ at various times. The right side indicates the rotating inner wall $\left(d^{*}=0\right)$.
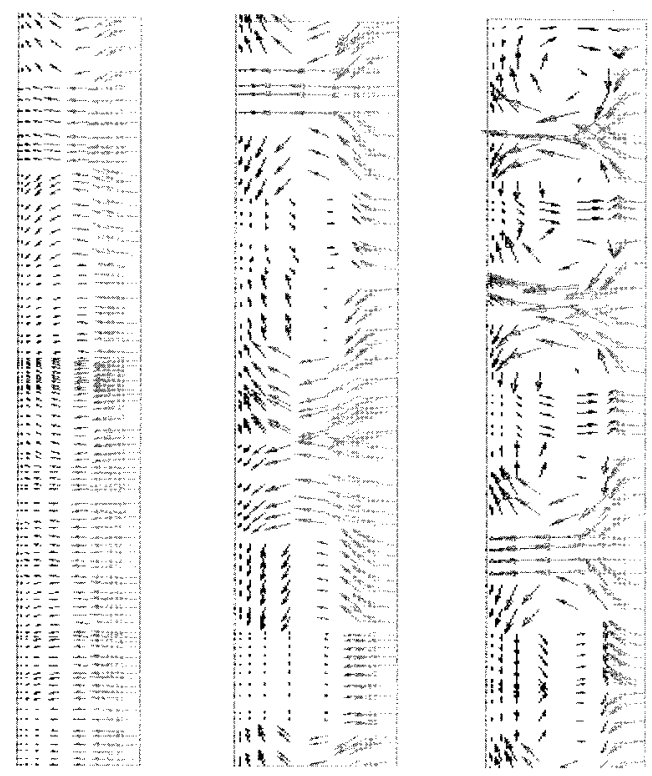

$\mathrm{y}^{*}=0.5-0.7$

$y^{*}=0.7-0.85$

$\mathrm{y}^{*}=0.85-1.0$

Figure 5. Velocity vectors shown in the radial plane for the ranges $\mathrm{y}^{*}=0.5-0.7, \mathrm{y}^{*}=0.7-0.85$ and $\mathrm{y}^{*}=0.85-1.0$ at $\mathrm{t}=330$ s. The right side indicates the rotating inner wall $\left(d^{*}=0\right)$.
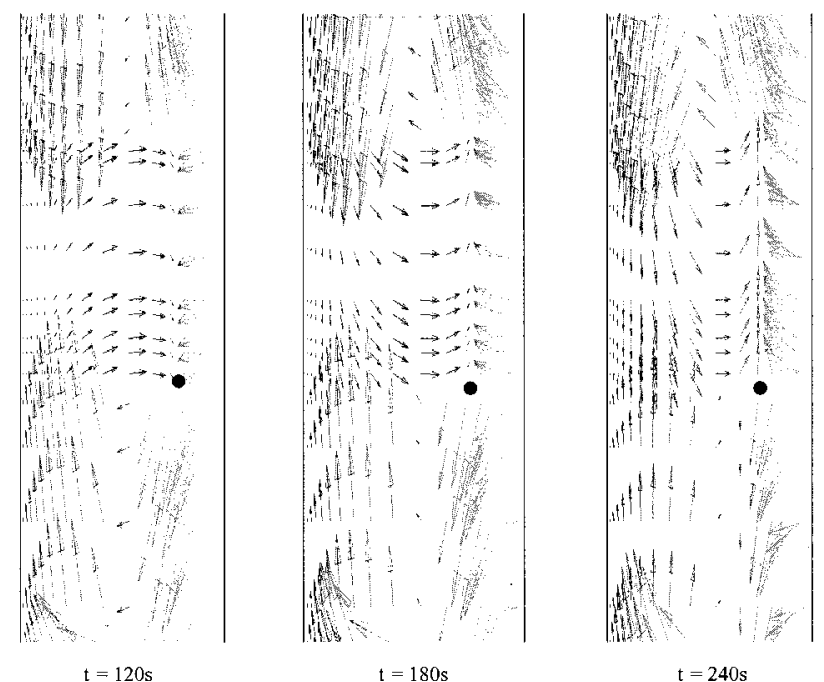

Figure 6. Internal boundary layer formation in Taylor-Couette flow and the presence of a saddle point. The velocity vectors in the radial plane for $y^{*}=0.855-0.885$ are shown. The right side indicates the rotating inner wall $\left(d^{*}=0\right)$.

of the flow in establshing the vortical roles. This instability and the associated mass transfer can be made more effective by initiating a secondary motion in which there is additional mass transfer between neighboring roles through the wavy vortex flow. This has been shown to be a strong function of Reynolds number by Werely and Lueptow, ${ }^{17}$ and it was reported that inter-role mass transfer is a maximum for the chosen geometry in their experiment for $\mathrm{Re}=253$. In the present set of computations, the same geometric parameters were chosen, including the aspect ratio, $(A R=L / d)$ of the reactor for the reference case. However, in the experiment, the starting protocol of the inner cylinder rotation was taken as quasi-static, and the reported results were recorded after the flow was allowed to develop for an additional $10 \mathrm{~min}$ after the attainment of the final rotation rate. In the present computations, the flow was started impulsively for the practical operation of the reactor in 
the shortest possible time period. For such an operation, the transient mass transfer depends strongly on the vortices that are formed at the initial times near the fixed end caps of the reactor. Because the flow inside the reactor is driven by the rotating inner cylinder and the rotation rates are low, it is quite adequate to consider the flow to be incompressible and to solve the governing Navier-Stokes equation in primitive variable form as

$$
\frac{\partial \overrightarrow{\mathrm{V}}}{\partial \mathrm{t}}+(\overrightarrow{\mathrm{V}} \cdot \nabla) \overrightarrow{\mathrm{V}}=-\frac{1}{\rho} \nabla p+v \nabla^{2} \overrightarrow{\mathrm{V}}
$$

and

$$
\nabla \cdot \overrightarrow{\mathrm{V}}=0
$$

Furthermore, isothermal conditions can be assumed for both the flow evolution and the chemical reaction calculations as the pollutant being oxidized into other products is present in trace amounts and the heat of reaction in photocatalytic reactions is usually negligible. $^{3}$

The following boundary conditions were used for the numerical simulations. With reference to Figure 1, on the inner cylinder surface, $r=r_{i}$

$$
\mathrm{V}_{\mathrm{r}}=\mathrm{V}_{\mathrm{y}}=0 \text { and } \mathrm{V}_{\theta}=\Omega_{\mathrm{i}} \mathrm{r}_{\mathrm{i}}
$$

where $\Omega_{\mathrm{i}}(\mathrm{t})$ is the instantaneous rotation rate of the inner cylinder. On the outer cylinder $\left(r=r_{0}\right)$ and the end caps $(\mathrm{y}=0$ and $\mathrm{L})$

$$
\mathrm{V}_{\mathrm{r}}=\mathrm{V}_{\mathrm{y}}=\mathrm{V}_{\theta}=0
$$

Because the simulation is for the impulsive start of rotation of the inner cylinder

$$
\begin{array}{ll}
\mathrm{t} \leq 0: & \Omega_{\mathrm{i}}(\mathrm{t})=0 \\
\mathrm{t}>0: & \Omega_{\mathrm{i}}(\mathrm{t})=\Omega
\end{array}
$$

The initial concentrations of phenol and SBB dye were taken as $100 \mathrm{ppm}$, while the initial mass fraction of $\mathrm{O}_{2}$ was taken as twice the stoichiometric requirement for the reaction. The oxidation reactions of phenol and SBB dye are given by

$$
\begin{gathered}
\mathrm{C}_{6} \mathrm{H}_{5} \mathrm{OH}+7 \mathrm{O}_{2} \rightarrow 6 \mathrm{CO}_{2}+3 \mathrm{H}_{2} \mathrm{O} \\
\mathrm{C}_{43} \mathrm{H}_{71} \mathrm{O}_{6} \mathrm{~N}_{3} \mathrm{~S}_{2}+64{ }_{1}^{1} \mathrm{O}_{2} \rightarrow \\
43 \mathrm{CO}_{2}+32 \mathrm{H}_{2} \mathrm{O}+2 \mathrm{H}_{2} \mathrm{SO}_{4}+3 \mathrm{HNO}_{3}
\end{gathered}
$$

respectively. Equations 2 and 3 are solved subject to the boundary conditions and initial conditions defined in eqs 4 and 5 within the annulus between the cylinders, as shown in Figure $1 \mathrm{a}$ and Table 1 . In computing the flow, no specific symmetry of the geometry was considered, i.e., full three-dimensional flow was solved even though the geometry is axis-symmetric. If the flow is indeed axis-symmetric, the solution would reflect this, and this viewpoint was adopted in solving and interpreting the solution. In the same way, the timeaccurate solution was sought, and if indeed there were a "steady state", the solution would indicate such. In the present case of computation, when the flow pattern is seen as symmetric, the results are shown in the radial-axial $(r-y)$ plane, as shown by the shaded region in Figure 1. For such symmetric profiles, the results are shown along

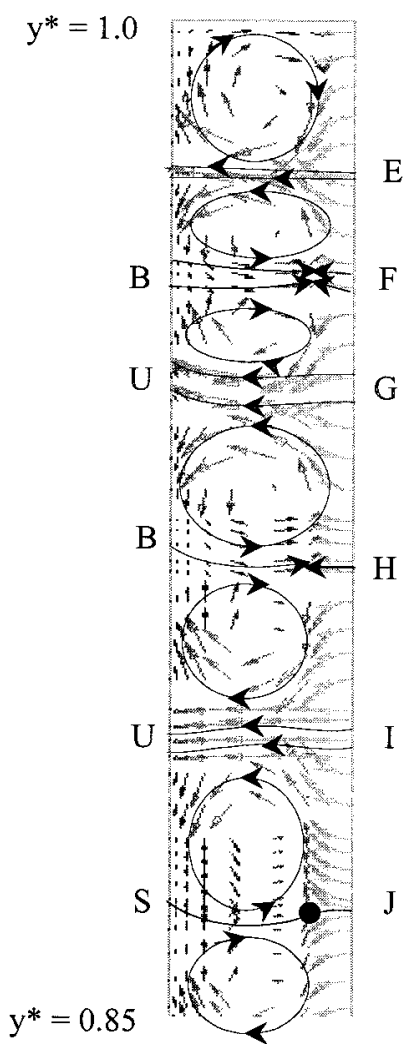

Figure 7. Flow structure of Taylor-Couette flow for an impulsive start at $\mathrm{t}=330 \mathrm{~s}$. The notations $\mathrm{U}, \mathrm{B}$, and $\mathrm{S}$ indicate unidirectional, bidirectional, and singular flow, respectively, and $\mathrm{E}-\mathrm{J}$ represent regions where such flows exist. The right side is the rotating inner wall $\left(d^{*}=0\right)$. indicates a saddle point.

the three sets of horizontal $\left(\mathrm{H}_{1}-\mathrm{H}_{3}\right)$ and vertical lines $\left(\mathrm{V}_{1}-\mathrm{V}_{3}\right)$ indicated in Figure $1 \mathrm{~b}$.

As al ready mentioned, the commercial software Fluent was used for the present simulation study. A brief description of the method is given below. The solver is based on solving eqs 2 and 3 in a sequential manner by a control-volume-based technique using the following three steps: First, the computing domain was discretized into discrete control volume using a computational grid. Then, the governing equations were time advanced in integral form for each computational cell to yield al gebraic equations for the discrete dependent variables, such as velocity components, pressure, and conserved scalars. Finally, the discrete equations were linearized into a set of al gebraic equations, which were solved to yield updated variables. For the discretization process, the familiar QUICK scheme was used for the momentum and species equations, as this is a higherorder accurate scheme with minimum numerical dissipation that is implicit with discretization.

In the search for the appropriate grid, the results obtained by the fine and coarse grids are first compared. In Figure 3, the grid convergence is shown with the hel $p$ of the plotted axial velocity contours for the finer and coarser grids at two different times. Although the plotted contours show some small differences at very early times (10 s), they are seen to disappear at later times $(t=30 \mathrm{~s})$. This shows that the coarser grid is adequate for the present investigation. Hence, unless stated otherwise, all of the reported results were obtained using the coarse grid. Similarly, using this coarse grid, we also performed calculations to investigate the effect of chemical reaction on flow evolution. In one case, only the fluid flow (no reaction) was 
(a)

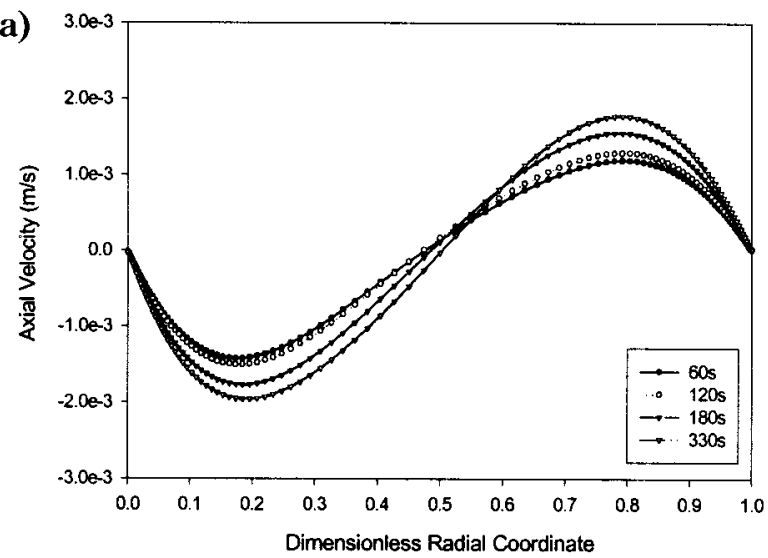

(b)

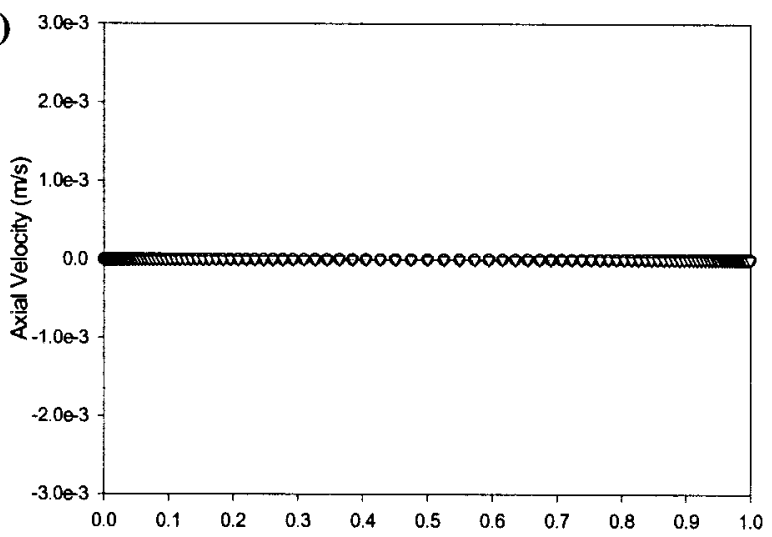

(c)

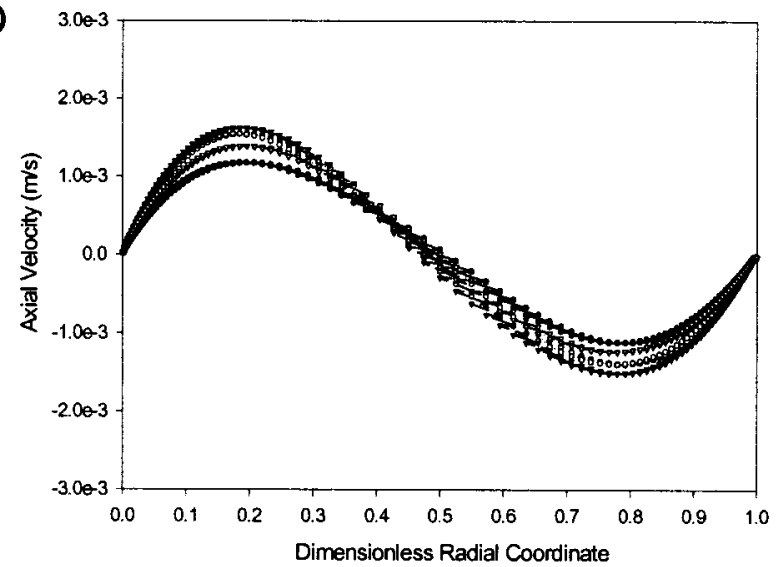

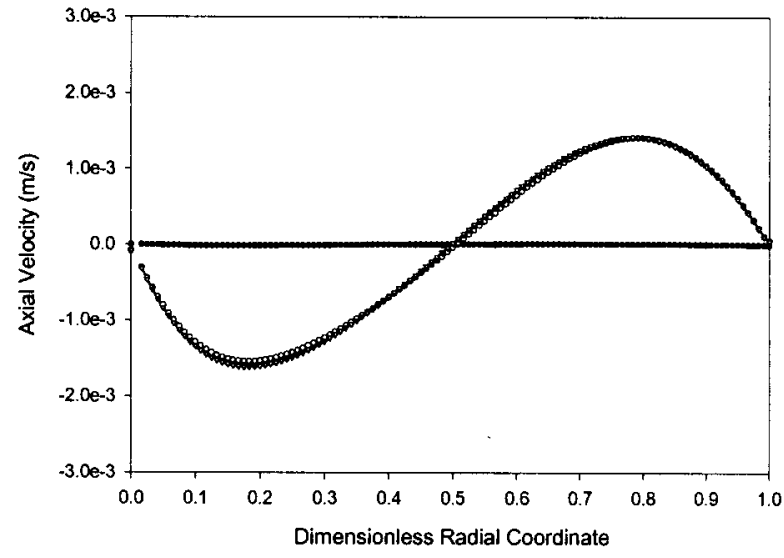
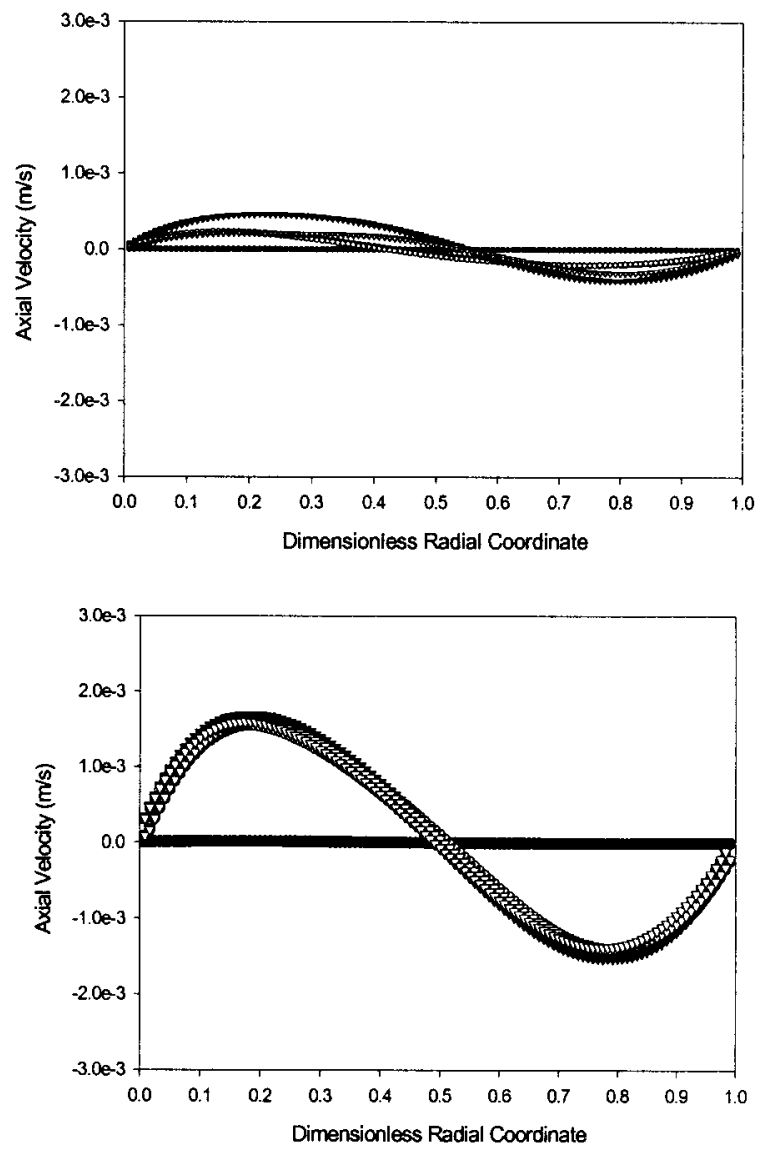

Figure 8. Change of axial velocity with dimensionless radial coordinate at different axial positions and times at (a) $\mathrm{H}_{1}$, (b) $\mathrm{H}_{2}$, and (c) $\mathrm{H}_{3}$. The figures on the left are for reactor configuration 1 , and those on the right are for configuration 2 . The right side indicates the rotating inner wall.

computed, whereas in the other case, the computations were carried out with the reaction proceeding concurrently. As the model component, phenol, was present at a low concentration (100 ppm), it was expected that the chemical reaction would not significantly alter the flow evolution. This was indeed found to be true, and the results are not shown here for brevity.

In Figure 4, the detailed vel ocity vectors are shown for the top half of the reactor $\left(y^{*}=0.5-1.0\right)$ in a given $y-r$ plane at different times, whereas in Figure 5, the velocity vector plots are shown at $\mathrm{t}=330 \mathrm{~s}$ when steady state has been reached. The segment between 50 and $70 \%$ of the reactor height is shown on the left, the segment between 70 and $85 \%$ is shown in the middle, and the remaining segment of the top half is shown on the right. One can see weak vortices forming near the upper part of the left segment. There are regions al ong the height where one can see a jetlike flow starting from the inner wall and moving toward the outer wall due to centrifugal action. In the segment for $y^{*}=0.7-0.85$, significant mixing of the fluid is noticeable a result of the formation of coherent vortices in part of the reactor. Once again, one can see jetlike flow from the inner to the outer cylinder, although the trajectories of the fluid particles are not strictly straight, as has been depicted in Figure 2. Moreover, no visi ble wall shear layer forms on the inner wall as has been shown in Figure 2c. In the segment for $y^{*}=0.85-1.0$, the velocity vectors clearly show recirculating rolls, although the axial lengths vary significantly because of end-wall effects. Also in this segment, apart from the jetlike regions, one can also see small axial regions where a flow is 

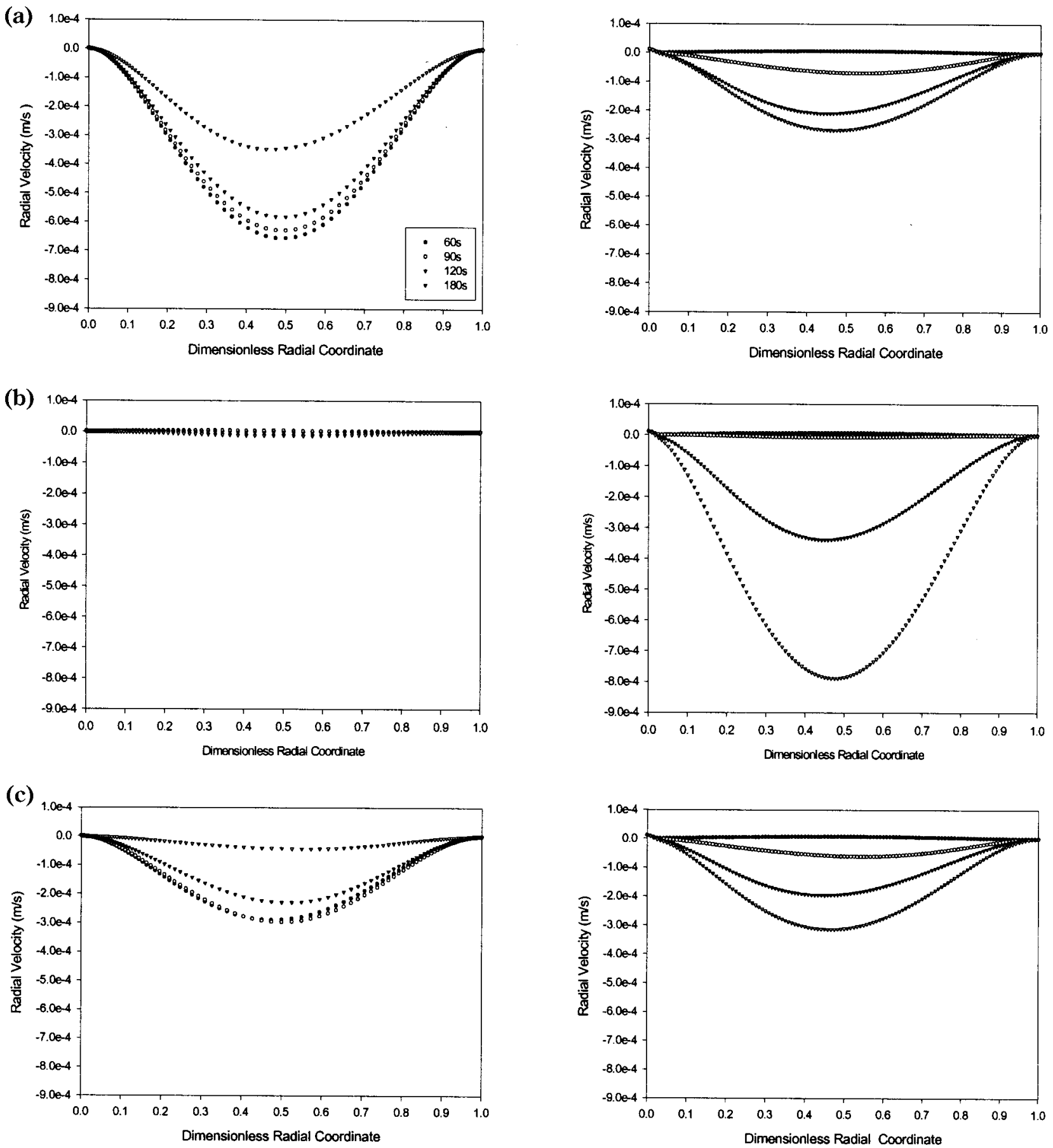

Figure 9. Change of radial velocity with dimensionless radial coordinate at different axial positions and times at (a) $\mathrm{H}_{1}$, (b) $\mathrm{H}_{2}$, and (c) $\mathrm{H}_{3}$. The figures on the left are for configuration 1 , and those on the right are for configuration 2 . The right side indicates the rotating inner wall.

established from the outer to the inner wall. However, this cannot cover the entire radial gap because of centrifugal force acting on the fluid particles near the inner wall. For the same reasons, one does not see a wall shear layer forming on the inner cylinder as has been shown in the sketch of Figure 2c. Instead, one would notice the formation of an internal layer after some time and the associated full saddle point inside the flow domain. In retrospect, it appears that, to resolve such internal layers, one should have a finer grid in the interior too. This is shown in Figure 6, where the velocity vectors are plotted over a limited segment between 85.5 and $88.5 \%$ of the reactor length. An internal band characterizes the formation of the internal layer where the flow is along the axis of the reactor. This is seen to originate at the saddle point, which is marked in the figure with a filled circle. The flow is seen to be axial in the opposite direction across the saddle point. Such vertical lines are nothing but the internal layer. The velocity vectors insi de the recirculating eddies show time-dependent behavior, although it is not as pronounced near the middle section of the reactor where the eddies are either very weak or not formed. All of these unsteady events would lead to an increase of mass exchange between adjacent fluid cells.

The flow structure for the chosen parameters of this computation with an impulsive start of the reactor is shown in Figure 7. This aspect is important in the 

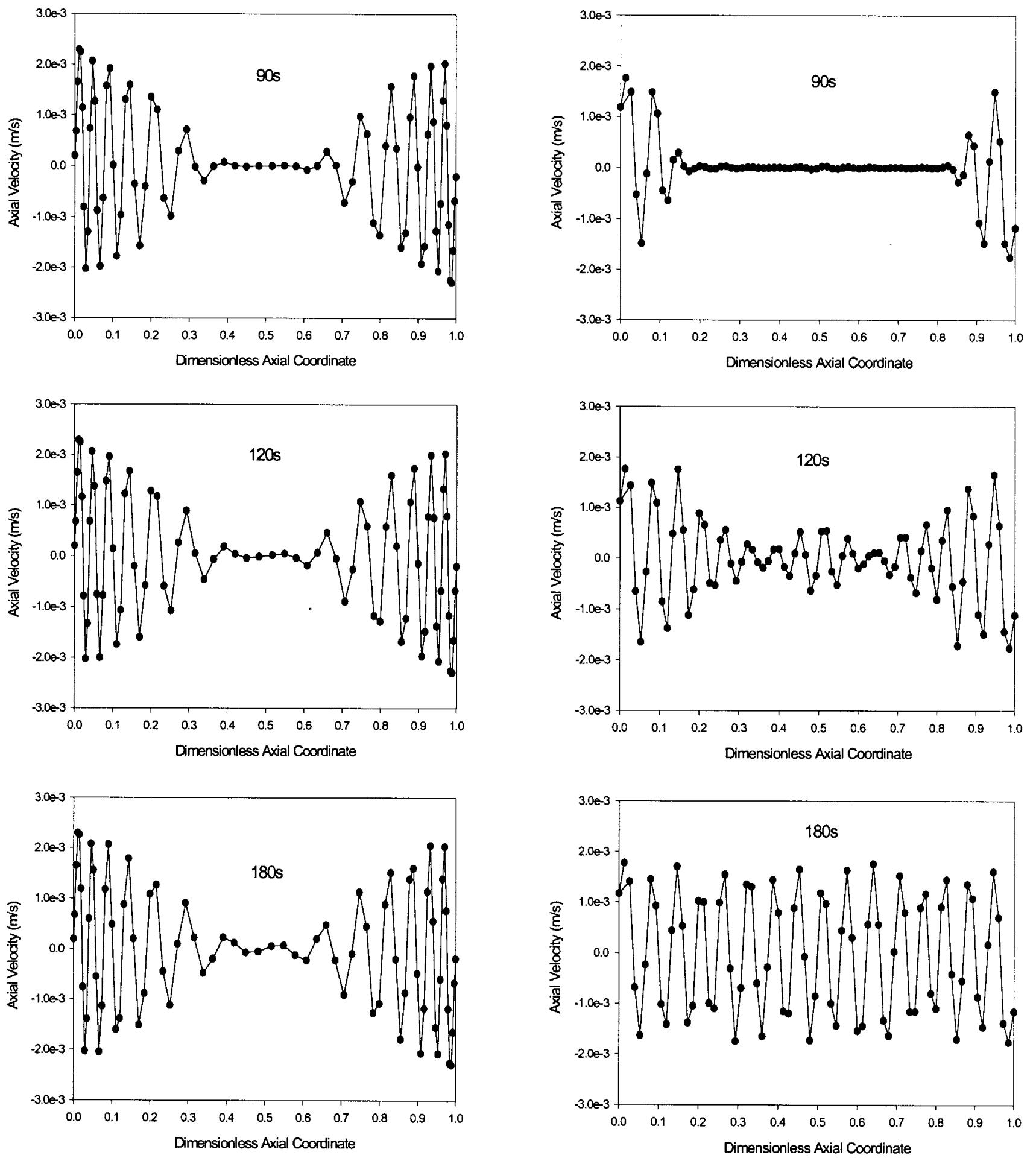

Figure 10. Change of axial velocity with dimensionless axial positions ( $\left.y^{*}=0-1.0\right)$ and time at location $\mathrm{V}_{1}$. The figures on the left are for configuration 1 while figures on right are for configuration 2.

context of nonunique flow evolution due to different initial conditions referred to in Andereck et al. ${ }^{16}$ The flow structure computed in this work is significantly different from what has been experimentally visualized by Werely and Lueptow, ${ }^{17}$ although the geometric parameters (aspect ratio) and Reynolds number are identical. This is due to the impulsive start of the present computed case as opposed to the quasi-static start in Werely and Lueptow, ${ }^{17}$ where the inner cylinder was accel erated from rest to its final value at the very slow rate of 0.3 Re per second. In Figure 7, the flow structure is shown for the segment $y^{*}=0.85-1.0$ at $t$
$=330 \mathrm{~s}$. The time is not important, as it was observed that the flow structure remains invariant beyond $\mathrm{t}=$ $180 \mathrm{~s}$. If one looks carefully in Figure 7, then one notices the jetlike flow structure between fluid cells. The portions marked by $\mathrm{E}, \mathrm{G}$, and I are the region where one can see unidirectional jets emerging from the inner wall and approaching the outer wall. This is the expected jet structure indicated in the sketch of Figure 2c. However, one can also notice the existence of bidirectional jets as marked by $\mathrm{F}$ and $\mathrm{H}$. A longer segment originating from the outer wall and moving toward the inner wall is met with a smaller segment 

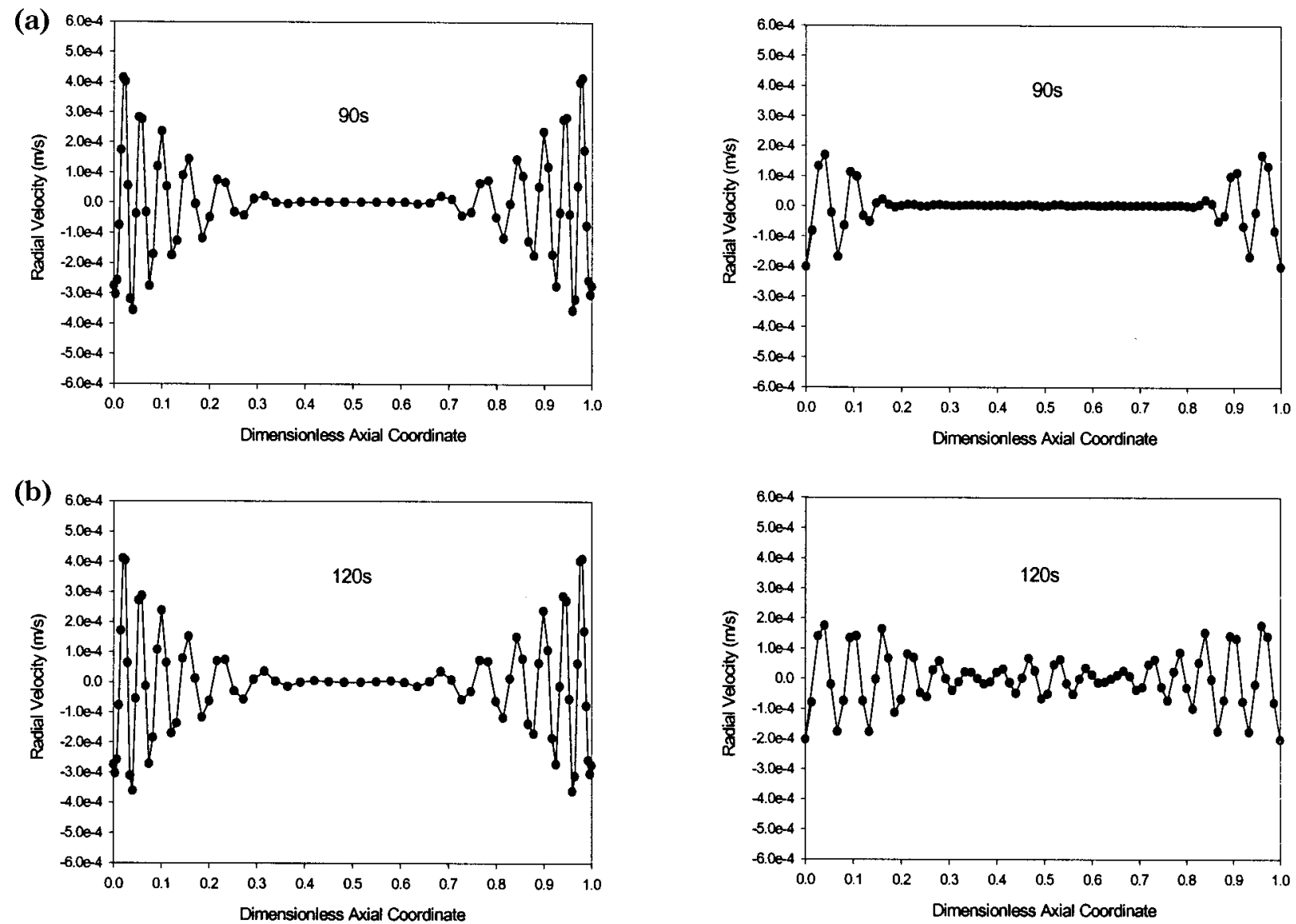

(c)
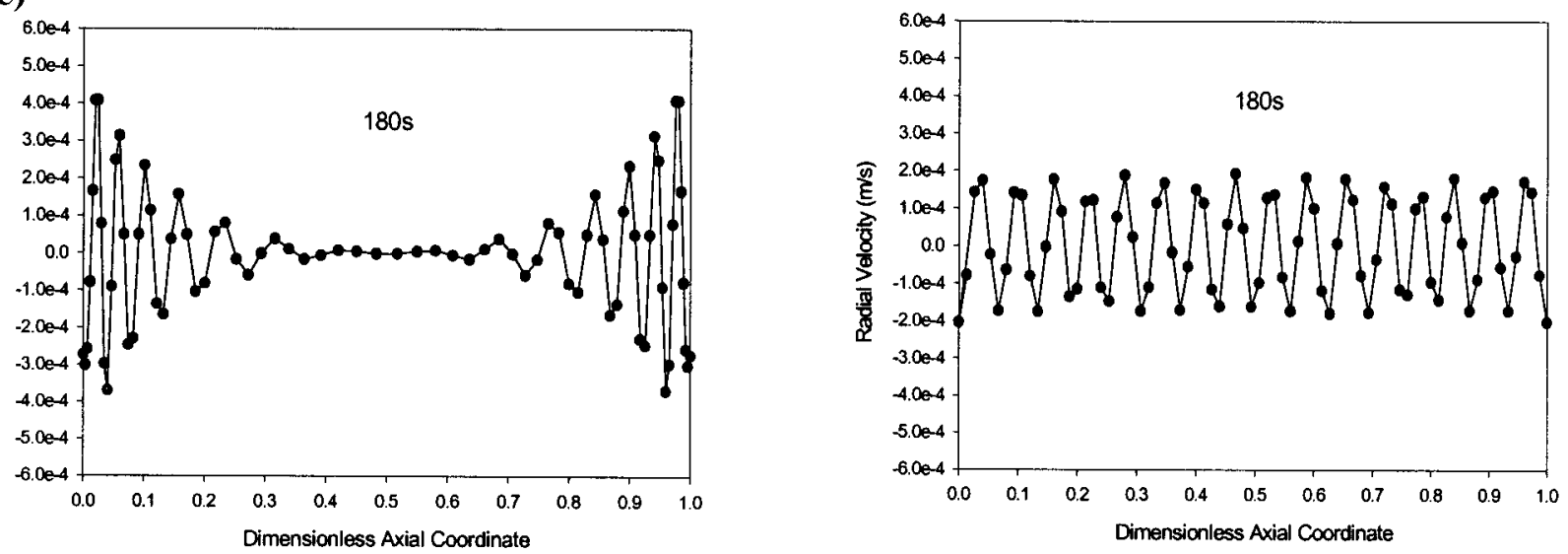

Figure 11. Change of radial velocity with dimensionless axial positions ( $y^{*}=0$ to 1.0 ) and time at location $\mathrm{V}_{1}$. Figures on the left are for configuration 1, and those on the right are for configuration 2.

where the flow is from the inner to outer wall. Then, we have marked a line J in the figure, which is the limiting case of the bidirectional jet when the width of the jet degenerates into a line and the merger line of the opposing jet becomes the saddle point. The notations $U, B$, and $S$ in the figure indicate the locations of unidirectional, bidirectional, and singular zones, respectively.

In Figures 8 and 9, respectively, the axial and radial velocities as functions of the dimensionless radial distance (annular gap) are plotted for various time instants at the observation stations $\mathrm{H}_{1}, \mathrm{H}_{2}$, and $\mathrm{H}_{3}$ of Figure $1 \mathrm{~b}$ for two different aspect ratios, configurations 1 and 2 of Table 1 . Because the middle section $\left(\mathrm{H}_{2}\right)$ is at the center $\left(y^{*}=0.5\right)$, the axial velocity is smallest in magnitude there as compared to the other two stations where the maximum vel ocity component is on the order $5-6 \%$ of the vel ocity scale. The results also indicate that the flow is not fully developed even at $t=330 \mathrm{~s}$, although the rate of change of flow has decreased significantly. For the middle plane $\left(\mathrm{H}_{2}\right)$, the axial and radial velocities demonstrate that the Taylor-Couette vortices move faster toward the middle part when the aspect ratio is lower (configuration 2). Figures 8 and 9 also indicate that there is an inflow of fluid toward the inner cylinder for all of the indicated times for the radial velocity whereas for the axial vel ocity component, inflow occurs at certain instants while outfl ow occurs at other instants within the annular gap. In addition, the magnitude of the adial velocity component is 1 order of magnitude lower than that of the axial velocity component. 

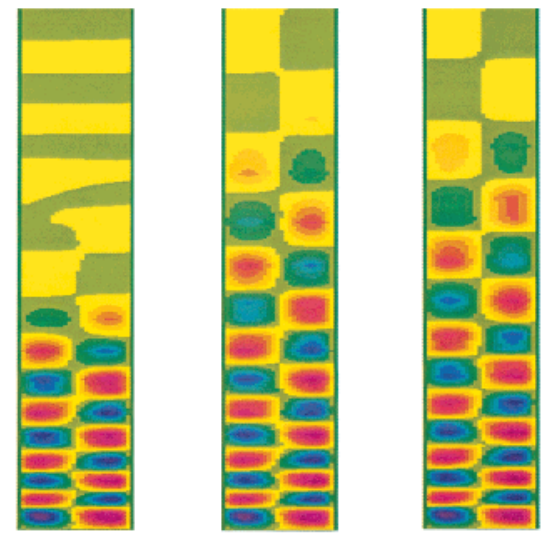

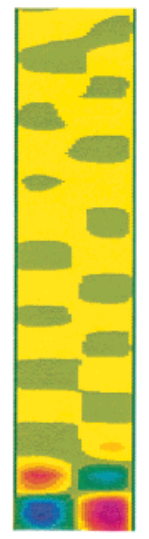

$60 \mathrm{~s}$

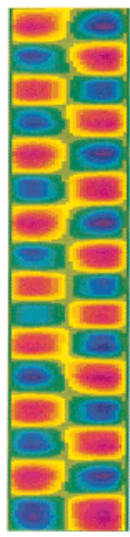

180 s

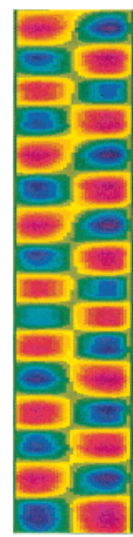

330 s
Figure 12. Flow development (axial velocity contour) for two reactor configurations. The top pictures are for configuration 1 , and the bottom pictures are for configuration 2. Only the bottom half of the reactor $\left(y^{*}=0-0.5\right)$ is shown. The right side indicates the rotating inner wall.

Table 3. Comparison of Performance of a Taylor Vortex Reactor (TVR) with that of a Classical Annular Reactor (CAR) for Photocatalytic Decomposition of Phenol

\begin{tabular}{|c|c|c|c|}
\hline & $\mathrm{CAR}^{18}$ & TVR 1 & TVR 2 \\
\hline volume of reactor, $\mathrm{m}^{3}$ & $3.48 \times 10^{-3}$ & $3.65 \times 10^{-3}$ & $3.96 \times 10^{-3}$ \\
\hline catalyst surface area, $\mathrm{m}^{2}$ & 0.18 & 0.116 & 0.116 \\
\hline $\mathrm{ICD},{ }^{\mathrm{a}} \kappa, \mathrm{m}^{2} / \mathrm{m}^{3}$ & 69 & 102 & 80 \\
\hline volume of liquid treated, $\mathrm{m}^{3}$ & $2.6 \times 10^{-3}$ & $1.136 \times 10^{-3}$ & $1.449 \times 10$ \\
\hline electrical energy input, W & 400 & 100 & 100 \\
\hline efficiency, ${ }^{b} \mathrm{~s}^{-1} \mathrm{~m}^{-3} \mathrm{~W}^{-1}$ & $1.0 \times 10^{-3}$ & $2.67 \times 10^{-2}$ & $1.44 \times 10^{-2}$ \\
\hline$\%$ increase in efficiency & 0 & 2570 & 1340 \\
\hline
\end{tabular}

a Illuminated catalyst density is defined as illuminated catalyst surface area $\left(\mathrm{m}^{2}\right)$ per unit volume of liquid treated $\left(\mathrm{m}^{3}\right)$ in the reactor (see ref 10). b Efficiency is defined as $50 \%$ pollutant converted per unit time per unit volume of liquid treated per unit electrical energy input.

In Figures 10 and 11 , respectively, the axial and radial velocity components are plotted al ong the dimensionless axial direction at observation stations $\mathrm{V}_{1}$ at three different times. At shorter times, the TaylorCouette vortices are formed at the ends, and as time progresses, more and more of them are formed, covering the middle part of the annulus. If, indeed, the vortices formed were like a plug flow, then the velocity component al ong this line would have been zero. Once again, we observe that vortices move faster toward the center when the aspect ratio is lower. The very fact that the velocity component alternates in sign indicates that the vortex centers not only execute axial waviness but also show significant radial motion. This was also observed
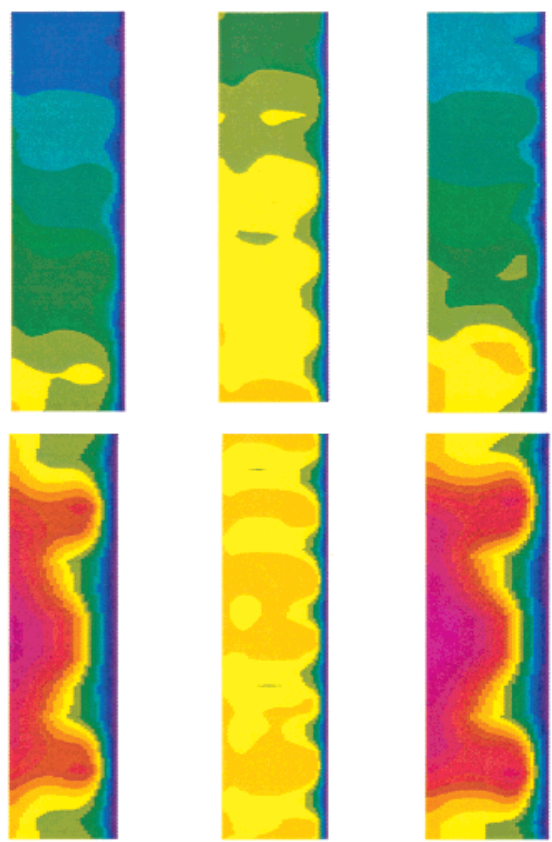

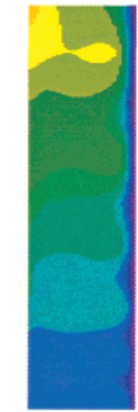

Configuration 1 Phenol

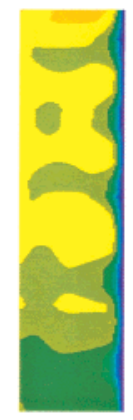

Configuration 2 Phenol

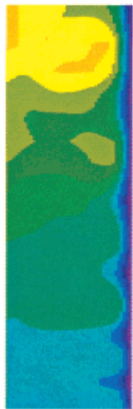

Configuration 1 SBB dye
Figure 13. Contour of photocatalytic degradation of phenol and SBB dye at $\mathrm{t}=330 \mathrm{~s}$. The entire reactor length is divided into the top $30 \%$, middle $40 \%$ and bottom $30 \%$. The scale of pollutant concentration is shown on the left $\left(1 \times 10^{-4} \equiv 100 \mathrm{ppm}\right)$.

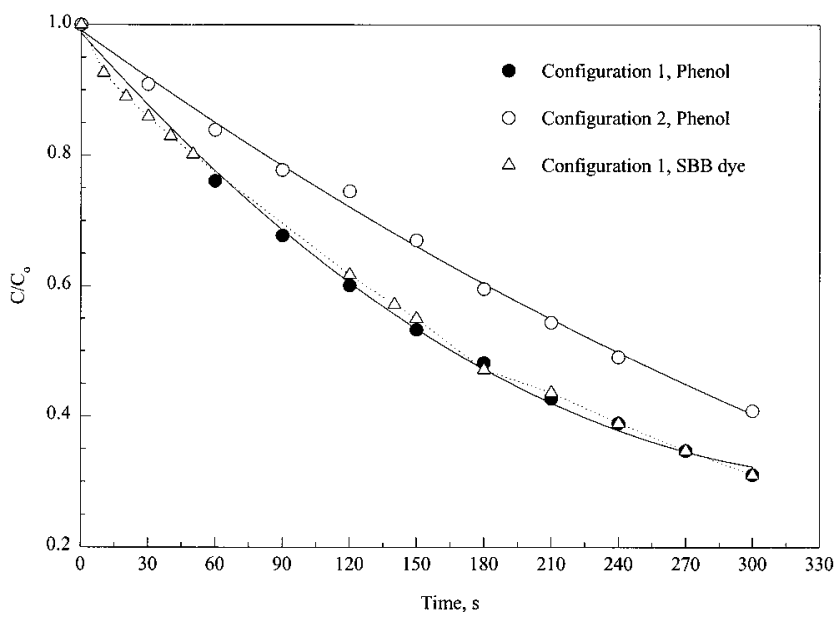

Figure 14. Photocatalytic degradation of phenol and SBB dye in reactor configurations 1 and 2 .

experimentally by Werely and Lueptow, ${ }^{17}$ where they showed such motions for all Reynolds numbers between 131 and 1221. However, it has to be noted that, in the present investigation, the flow is started impulsively and not accelerated quasi-statically and the flow is not completely fully devel oped. This aspect is clearly dem- 
Table 4. Comparison of Performance of a TVR with that of a Classical Annular Reactor (CAR), a Slurry Reactor (SR), a Multiple Tube Reactor (MTR), and a Tube Light Reactor (TLR) for Photocatalytic Decomposition of SBB Dye

\begin{tabular}{llllll}
\hline & \multicolumn{1}{c}{ CAR $^{18}$} & \multicolumn{1}{c}{ SR $^{18}$} & \multicolumn{1}{c}{ MTR $^{9}$} & \multicolumn{1}{c}{ TLR $^{10}$} \\
\hline volume of reactor, $\mathrm{m}^{3}$ & $3.48 \times 10^{-3}$ & $3.4 \times 10^{-3}$ & $1.23 \times 10^{-3}$ & $5.36 \times 10^{-3}$ & $3.65 \times 10^{-3}$ \\
catalyst surface area, $\mathrm{m}^{2}$ & 0.18 & 3.7 & 0.51 & 0.15 & 0.116 \\
ICD, $^{\mathrm{a}}, \mathrm{m}^{2} / \mathrm{m}^{3}$ & 69 & 6139 & 1087 & 618 & 102 \\
volumetric flow rate, $\mathrm{m}^{3} / \mathrm{s}$ & $8.42 \times 10^{-5}$ & Batch operation & $3.0 \times 10^{-5}$ & $1.67 \times 10^{-5}$ & $\mathrm{Batch}_{\text {operation }}$ \\
volume of liquid treated, $\mathrm{m}^{3}$ & $2.6 \times 10^{-3}$ & $6.0 \times 10^{-4}$ & $4.69 \times 10^{-4}$ & $2.43 \times 10^{-4}$ & $1.136 \times 10^{-3}$ \\
electrical energy input, $\mathrm{W}$ & 400 & 960 & 40 & 126 & 100 \\
efficiency, $\mathrm{s}^{-1} \mathrm{~m}^{-3} \mathrm{~W}^{1-}$ & $9.43 \times 10^{-4}$ & $3.1 \times 10^{-3}$ & $1.18 \times 10^{-2}$ & $1.67 \times 10^{-2}$ & $2.67 \times 10^{-2}$ \\
\% increase in efficiency & 0 & 229 & 1157 & 1668 & 2730
\end{tabular}

a Illuminated catalyst density is defined as illuminated catalyst surface area $\left(\mathrm{m}^{2}\right)$ per unit volume of liquid treated $\left(\mathrm{m}^{3}\right)$ in the reactor (see ref 10 ). ${ }^{b}$ Efficiency is defined as $50 \%$ pollutant converted per unit time per unit volume of liquid treated per unit electrical energy input.

onstrated in the contour plots of the axial velocity component al ong the entire length of the reactor shown in three segments in Figure 12. The figure shows that flow develops slowly for configuration 2 (lower aspect ratio), but vortices move faster toward the center of the reactor.

To analyze the performance of the TVR, phenol and SBB dye were considered as pollutants present in water with initial concentrations of 100 ppm. The photocataIytic degradation rates for phenol and SBB dye are given in Table 2. The degradation rate of the SBB dye is about 400 times slower than that of phenol. In Figure 13, the pollutant mass fraction contours are shown at $t=240$ $s$ for the photocatalytic degradation of phenol in reactor configurations 1 and 2 and of SBB dye in the reference reactor. A close scrutiny of Figure 12, where the velocity vectors are shown, explains the mass fraction contour plots of the pollutants in Figure 13. It reveals that the reaction is faster at either end where well-developed vortices are present, whereas the degradation rates are slower where Taylor-Couette vortices are not wellformed. This type of flow evolution directly influences the photocatalytic oxidation of pollutant and can be seen in Figure 13. The reaction takes place only in the shear layer of the inner cylinder where no Taylor-Couette vortices are present. Pollutant degradation integrated over the full reactor volume is shown as a function of time in Figure 14. This pl ot clearly indicates the role of Taylor-Couette vortices in enhancing the rate of pollutant degradation. The figure clearly illustrates that the photocatalytic reactions are diffusion-controlled, as the rate of degradation of SBB dye is same as that of phenol even though the rate constant for the dye is about 400 times lower than that for phenol. The overall reaction is primarily controlled by mass transfer and not by intrinsic kinetics. The figure also illustrates that the degradation rate for phenol in TVR 2 is slower than that in the reference-configuration reactor (TVR 1). This is due to two factors. First, even though, for reactor configuration 2, the vortices move faster toward the center from the end caps, the magnitude of the vortices is smaller. Second, the amount of catalyst per unit volume of liquid treated (the parameter, $\kappa$ ) is lower in reactor 2. The volume of liquid treated in TVR 2 is $27.5 \%$ more than that of TVR 1 . Figures 13 and 14 also indicate the possibility of operating the reactor in the transient mode by periodically switching the reactor on and off because, during the transient phases, the reaction proceeds at a rapid rate because of the vortices near the end walls. Table 3 compares the performance of a classical annular reactor $(C A R)^{18}$ with those of the two TVR configurations considered in this work for the photocatalytic degradation of phenol, while Table 4 compares the performance of a TVR with those of a
$\mathrm{CAR},{ }^{18}$ a slurry reactor (SR), ${ }^{18}$ a multiple tube reactor (MTR), ${ }^{9}$ and a tube light reactor (TLR) ${ }^{10}$ for the photocatalytic degradation of SBB dye. The table clearly illustrates the efficiency of the TVR over the other reactors. One can observe an increase in efficiency of the TVR of $60 \%$ over the tube light reactor ${ }^{10}$ and $125 \%$ over the multiple tube reactor. ${ }^{9}$

\section{Conclusions}

Heterogeneous photocatalysis on semiconductor particles has been shown to be an effective means of removing toxic organic pollutants from water. Earlier experimental studies of our group on catalyst-coated tube bundles, novel immersion-type lamps and rotating tube bundles revealed that photocatalytic reaction is mainly diffusion-controlled. The reaction occurs at the fluid-catalyst interface, and in most cases, the overall rate of reaction is limited by the mass transport of the pollutant to the catalyst surface. In our earlier studies, we enhanced mass transfer by increasing the mixing of fluids through turbulence and introduction of baffles. In this work, a new photocatalytic reactor design is considered where an increase in conversion for the in situ degradation of toxic pollutants is achieved through introduction of fluid flow instability. The new idea of using fluid flow instability to increase the reaction yield throughout the reactor volume is shown to produce effective pollutant reduction. In this work, we considered unsteady Taylor-Couette flow between two coaxial cylinders, where the inner cylinder (coated with $\mathrm{TiO}_{2}$ catalyst) is rotated to achieve the desired instability. The main advantage of considering this particular type of flow pattern as our design consideration is its creation of wavy vortex flow in the laminar-flow regime. Significant transfer of fluid between neighboring vortices occurs in a cyclic fashion along a certain wave, and net axial flow also occurs in which fluids wind around the vortices. Two different reactor configurations were considered that depict two different aspect ratios of the reactor. The flow fields were calculated for a constant Reynol ds number, and it was observed that, in the lower aspect rati o case, vortices move faster toward the center although the magnitudes are smaller. Two different pollutants were considered, namely, phenol and a textile dye, that have two very different rate constants. The rate of photocatalytic oxidation increased dramatically. It was observed that the reaction is completely diffusioncontrolled, as both phenol and SBB dye degraded at the same rate. The Taylor vortex reactor was found to be an excellent reactor, as the increase in reactor performance efficiency was found to be 60 and $125 \%$ over those of a tube light reactor ${ }^{10}$ and a multiple tube reactor, ${ }^{9}$ respectively. 


\section{Notation}

$\mathrm{AR}=$ aspect ratio

$\mathrm{d}=$ annular gap, $\mathrm{m}$

$\mathrm{L}=$ length, $\mathrm{m}$

$\mathrm{r}=$ radius, $\mathrm{m}$; radial direction, $\mathrm{m}$

$\mathrm{Re}=$ Reynolds number

$\mathrm{t}=$ time, $\mathrm{s}$

$\mathrm{Ta}=$ Taylor number

$\mathrm{V}=$ velocity, $\mathrm{m} / \mathrm{s}$

\section{Symbols}

$\kappa=$ illuminated catalyst density, $\mathrm{m}^{2} / \mathrm{m}^{3}$

$\Omega=$ rotation rate, $\mathrm{rad} / \mathrm{s}$

$\rho=$ density, $\mathrm{kg} / \mathrm{m}^{3}$

$v=$ kinematic viscosity, $\mathrm{m}^{2} / \mathrm{s}$

Subscripts/ Superscripts

$\mathrm{i}=$ inner

$\mathrm{o}=$ outer

\section{Literature Cited}

(1) Chen, D. W.; Sivakumar, M.; Ray, A. K. Semiconductor Photocatalysis in Environmental Remediation. Dev. Chem. Eng. Min. Process. 2000, 8 (5/6), 505.

(2) Chen, D. W.; Ray, A. K. Photodegradation Kinetics of 4-Nitrophenol in $\mathrm{TiO}_{2}$ Aqueous Suspensions. Water Res. 1998, 32, 3223.

(3) Chen, D. W.; Ray, A. K. Photocatalysis Kinetics of Phenol and Its Derivatives over UV-Irradiated $\mathrm{TiO}_{2}$. Appl. Catal. B: Environ. 1999, 23, 143.

(4) Chen, D. W.; Ray, A. K. Removal of Toxic Metal Ions in Wastewater by Semiconductor Photocatalysis. Chem. Eng. Sci. 2001, 56 (4), 1561.

(5) Ray, A. K.; Beenackers, A. A. C. M. A Novel Swirl Flow Reactor for Kinetic Studies of Semiconductor Photocatalysis. AIChE J . 1997, 43, 2571.

(6) Mukherjee, P. S.; Ray, A. K. Major Challenges in the Design of a Large-Scale Photocatalytic Reactor for Water Treatment. Chem. Eng. Technol. 1999, 22, 253.

(7) Periyathamby, U.; Ray, A. K. Computer Simulation of a Photocatalytic Reactor Using Distributive Computing. Chem. Eng. Technol. 1999, 22 (10), 881.

(8) Chen, D. W.; Li, F.; Ray, A. K. Effect of Mass Transfer and Catalyst Layer Thickness on Photocatalytic Reaction. AIChE J . 2000, 46 (5), 1034.

(9) Ray, A. K. Design, Modeling and Experimentation of a New Large-Scale Photocatalytic Reactor for Water Treatment. Chem. Eng. Sci. 1999, 54 (16), 3113.

(10) Ray, A. K.; Beenackers, A. A. C. M. A Novel Photocatalytic for Water Purification. AIChE J. 1998, 44, 477.

(11) Sczechowski, J. G.; Koval, C. A.; Noble, R. D. A Taylor Vortex Reactor for Heterogeneous Photocatalysis. Chem. Eng. Sci. 1995, 50 (20), 3163.

(12) Taylor, G. I. Stability of a Viscous Liquid Contained between Two Rotating Cylinders. Philos. Trans. R. Soc. 1923, A223, 289 .

(13) Lord Rayleigh. On the Dynamics of Revolving Fluids. Scientific papers, Cambridge, England, 1920; Vol. 6, pp 447-453.

(14) Chandrasekhar, S. Hydrodynamics and Hydromagnetic Stability; Oxford University Press: New York, 1961; pp 273-340.

(15) Burkhalter, J . E.; Koschmieder, E. L. Steady Supercritical Taylor Vortex Flow. J . Fluid Mech. 1973, 58, 547.

(16) Andereck, C. D.; Liu, S. S.; Swinney, H. L. Flow Regimes in a Circular Couette System with Independently Rotating Cylinders. J . Fluid Mech. 1986, 164, 155.

(17) Werely, S. T.; Lueptow, R. M. Spatio-temporal Character of Non-Wavy and Wavy Taylor Couette Flow. J . Fluid Mech. 1998, 364, 59.

(18) Assink, J . W.; Koster, T. P. M.; Slaager, J . M. FotokataIytische oxydatie voor afval water-behandeling; Report Reference No. 93-137; I nstituut voor Milieu en Energie-technologie (TNO): Apeldoorn, The Netherlands, 1993.

Received for review December 20, 2000 Revised manuscript received J une 6, 2001 Accepted J une 6, 2001

IE001120| 